\title{
THE CHIRPED-PULSE INVERSE FREE-ELECTRON LASER
}

\author{
A. L. Troha ${ }^{\dagger}$, F. V. Hartemann, D. J. Gibson, E. C. Landahl, LLNL, Livermore, CA 94550, USA
}

\begin{abstract}
We propose a new accelerating concept, the chirpedpulse inverse free-electron laser (CPIFEL). We study the inverse free-electron laser (IFEL) accelerator both theoretically and computationally, and show that by using a femtosecond (fs), ultra-high-intensity drive laser pulse, the IFEL interaction bandwidth and accelerating gradient are increased, thus yielding large energy gains. Using a chirped pulse and negative dispersion focusing optics allows one to take advantage of the laser bandwidth and produce a chromatic line focus, maximizing the gradient. Combining these ideas results in a compact vacuum laser accelerator capable of accelerating picosecond (ps) electron bunches with a high gradient $(\mathrm{GeV} / \mathrm{m})$ and very low energy spread.
\end{abstract}

\section{INTRODUCTION}

The inverse free-electron laser accelerator (IFELA) is a promising concept in the development of a true vacuum laser acceleration device. The first experiments demonstrating energy gain using an IFELA were performed at Columbia University [1] and Brookhaven National Laboratory [2]. The IFELA is a favorable device for laser acceleration because the interaction occurs in a vacuum, away from boundaries, thus permitting a large interaction region; since a plasma is not employed as the accelerating medium, this resolves a number of problems (including plasma instabilities, nonlinear laser propagation, shot-toshot reproducibility, and the extremely small accelerating potential well) which plague plasma-based accelerator schemes. The IFEL wiggler is apt to afford good focusing and electron-beam optics and transport, thus possibly producing the high-quality electron beams required for advanced light sources, biomedical applications, and the Next Linear Collider. However, the typical IFELA scheme suffers from the phase-slippage of the trapped electron with respect to the drive laser wave. As the electron gains energy, the free-electron laser (FEL) resonance condition [3] cannot be maintained, and the electron attains a maximum energy dictated by the FEL interaction bandwidth. This problem can be amended by either using a tapered wiggler period and/or amplitude [2, 4] or, equivalently, by using a chirped drive laser pulse. We use the latter approach, since it is easier to adjust the timing of the electron bunch and the laser pulse than it is to bring the electrons and laser pulse together at a precise position along the wiggler. One should note that at present, IFELA gradients are comparable to those possible with high-frequency radio-frequency (rf) systems (roughly up to $100 \mathrm{MeV} / \mathrm{m}$ ).

†altroha@ieee.org
We examine the IFEL interaction in which fs, TW-class drive laser pulses generated by tabletop systems using chirped pulse amplification (CPA) are used [5]. For such fs laser pulses, the IFEL interaction bandwidth is much wider than it is for lower-intensity drive pulses with durations longer than a nanosecond. The FEL resonance condition indicates that when the electron slips over one laser optical cycle, it also propagates over one wiggler period; thus, for fs pulses, the wiggler interaction region is extremely short, and the IFEL resonance bandwidth is very wide. The electron energy now increases significantly before the IFEL interaction detunes and saturates; thus the CPIFEL yields a high accelerating gradient $(>1 \mathrm{GeV} / \mathrm{m})$. Hence, the IFEL interaction physics will be different for broadband (fs) drive laser pulses near the relativistic intensity regime ( $>10^{17} \mathrm{~W} / \mathrm{cm}^{2}$ for optical wavelengths).

We describe how using a chirped laser pulse allows the FEL resonance condition to be maintained beyond the usual dephasing limit, thus improving the electron energy gain, and how the ultrashort, high-intensity laser pulses generated using CPA permits the design of an IFELA with very high accelerating gradients $(>1 \mathrm{GeV} / \mathrm{m})$, in contrast to the previous long-pulse approaches. Another limitation in IFELA's is the diffraction of the drive laser pulse. This is alleviated by using the ultra-wide bandwidth of the chirped laser pulse: Negative dispersion focusing optics produce a chromatic line focus, where long wavelengths are focused first, while the shorter wavelengths, which maintain the FEL resonance condition at higher energies, are focused further along the interaction region. A ray-tracing study of this chromatic line focus was made. It was demonstrated numerically that the accelerating IFEL bucket is very wide compared to the plasmabased schemes. We found that for a 1 ps FWHM (fullwidth at half-maximum) Gaussian electron bunch, and a $1-\mathrm{cm}$ period wiggler, the IFEL energy spread is $<0.9 \%$.

\section{ONE-DIMENSIONAL CPIFEL THEORY}

A brief discussion of this novel concept can be given by considering the FEL resonance condition [3], $\lambda$ $\approx \lambda_{w}\left(1+A_{w}^{2}\right) / 2 \gamma^{2}$, where $\lambda$ is the drive laser wavelength, $\lambda_{w}$ is the wiggler period, $A_{w}=e B_{w} \lambda_{w} / 2 \pi m_{0} c$ is the normalized vector potential of the wiggler, and $\gamma$ is the electron energy. In this equation, the laser intensity is assumed to be small enough so that radiation pressure is negligible, i. e., the normalized vector potential of the laser satisfies the condition $A_{0}^{2}=\left(e E_{0} / \omega_{0} m_{0} c\right)^{2} \ll 1$. The resonance cannot be maintained as the electron energy 
increases during the IFEL acceleration process, since the effective mass of the electron increases, which changes the resonance condition for the FEL pendulum equation. Thus, the process stagnates, limiting the maximum possible energy gain, and it can reverse direction, going into the FEL mode where the electron looses energy to the electromagnetic field. The higher the accelerating gradient, the faster dephasing occurs; but, for ultrashort laser pulses, this is balanced by the increased interaction bandwidth. To alleviate this problem, we use a chirped pulse, where the drive laser wavelength, $\lambda$, decreases as the electron gains energy, thus maintaining resonance beyond the above-mentioned saturation length. Such chirped, ultrahigh-intensity laser pulses are easily produced using CPA, and tabletop systems with high repetition rates $(20-1000 \mathrm{~Hz})$ are now available.

\subsection{General Results of Numerical Simulations}

A detailed theoretical analysis of the CPIFEL interaction has been presented elsewhere [6], and we cover the pertinent results of that work here. For reference, the vector potential of the laser is modeled by a circularly polarized plane wave with a linear chirp, $\alpha$,

$$
\mathbf{A}_{\ell}(\phi)=A_{0} g(\phi)\left[\hat{x} \sin \left(\phi+\alpha \phi^{2}\right)+\hat{y} \cos \left(\phi+\alpha \phi^{2}\right)\right],
$$

where we employ the phase of the traveling laser wave, $\phi=k_{\mu} x^{\mu}=t-z$. In these equations, length is measured in units of the central laser wavelength, $1 / k_{0}=\lambda_{0} / 2 \pi$, and time in units of the laser frequency, $1 / \omega_{0}$. The pulse envelope is chosen as $g(\phi)=\sin ^{2}[\phi / \Delta \phi]$, which closely approximates a Gaussian near the maximum, and has a finite duration. Also, $\Delta \phi$ is the pulse FWHM, measured in units of $1 / \omega_{0}$, and $A_{0}$ is the amplitude of the laser pulse measured in units of $m_{0} c / e$. The helical wiggler field is given by the usual approximation,

$$
\mathbf{A}_{w}(z)=A_{w}\left[\hat{x} \sin \left(k_{w} z\right)+\hat{y} \cos \left(k_{w} z\right)\right],
$$

where $k_{w}=\lambda_{0} / \lambda_{w}$ is the wiggler wave number.

As an example, we ran the code using the following values: $A_{0}=0.2$ for the normalized vector potential of the drive laser pulse, a 10 -fs pulse duration, a central laser wavelength of $\lambda_{0}=0.8 \mu \mathrm{m}, B_{w}=2 \mathrm{kG}$ for the wiggler field, a wiggler period of $\lambda_{w}=1 \mathrm{~cm}$, an injection energy of $\gamma_{0}=65$, and a resonant IFEL energy of $\gamma^{*}=80$. The results are shown in Fig. 1 for both an unchirped laser pulse, and an optimally chirped laser pulse, where $\alpha=0.007$. In the first case, the electron detunes away from the IFEL resonance, and exits the interaction region with an energy gain corresponding to an average acceleration gradient of $0.343 \mathrm{GeV} / \mathrm{m}$. This value is quite high since we are using an ultrashort laser pulse with a high focused intensity, equivalent to $8.55 \times 10^{16} \mathrm{~W} / \mathrm{cm}^{2}$ for the parameters given above. Any attempt to decouple the electron at its peak energy will result in a severe loss of beam quality. In the second case, however, the IFEL resonance is maintained throughout the interaction region, and the electron energy now corresponds to an average gradient of $0.567 \mathrm{GeV} / \mathrm{m}$, or a $65 \%$ increase over the unchirped case. Also, in this case, no extraction scheme is required, thus maintaining the accelerated beam quality.

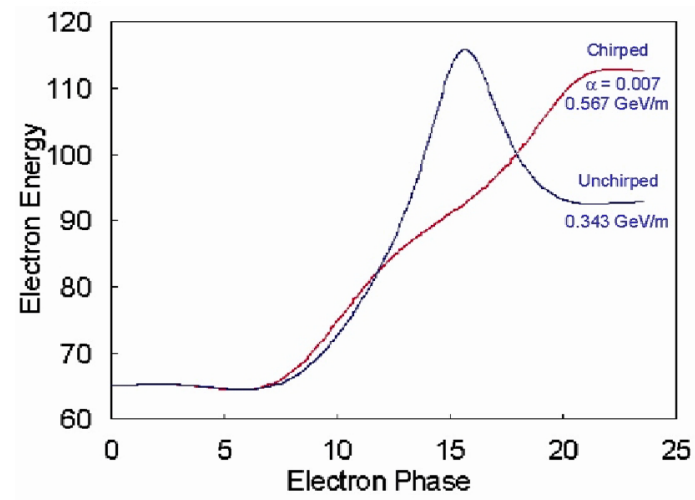

Figure 1. Electron normalized energy as a function of its phase within the drive laser pulse. $A_{0}=0.2,10 \mathrm{fs}$ FWHM,

$$
\lambda_{0}=0.8 \mu \mathrm{m}, B_{w}=2 \mathrm{kG}, \lambda_{w}=1 \mathrm{~cm}, \gamma_{0}=65 \text {. }
$$

\subsection{Chromatic Line Focus}

The intensity of the laser along the focal region can be maximized by using an optical scheme which produces a chromatic line focus. The long-wavelength part of the pulse will be focused closer to the optics, with the shorter wavelengths focused further down the propagation axis. This can be accomplished in various ways, using either reflective, refractive, or diffractive optics. This approach is expected to relax the drive laser energy requirement by an order of magnitude. We have performed a preliminary ray-tracing study of the chromatic line focus using Optica [7]; the results are shown in Fig. 2, where the optical setup is presented schematically (left), and the resulting focal spot position is graphed as a function of the wavelength (right). The focusing system consists of
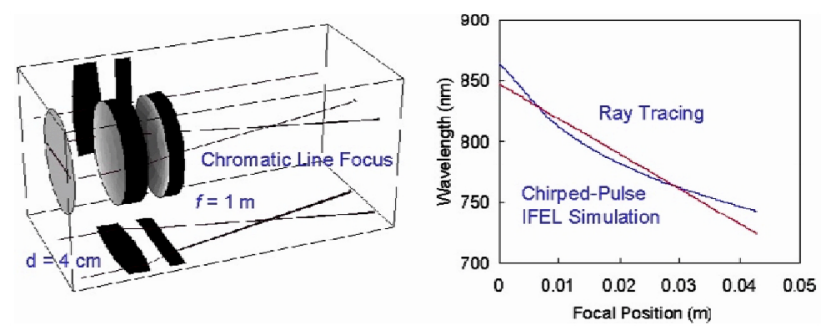

Figure 2. Optica ray-tracing results for a chromatic line focus; details are given in the text.

a lens pair, including a biconvex lens made of flint glass, and a plano-concave lens made of crown glass. The group velocity dispersion (GVD) introduced by the doublet can be recompensated for in the CPA stretcher, following the approach by Barty et al. [8]. The chromatic line focus is 
shown in Fig. 2 (right) and compared to the CPIFEL simulation; the results agree well to first-order, and demonstrate the validity of this approach. The design of higher-order line focusing optics will be performed in the near future, where a number of important issues will be addressed, including GVD and $B$-integral effects in the chromatic focusing doublet. The three-dimensional (3-D) modeling of the chromatic line focus for an ultra-wideband, high-intensity laser pulse will be achieved by modifying our paraxial propagator method [9], which includes axial electromagnetic field components and satisfies the gauge condition exactly. These 3-D field distributions will be used in conjunction with a relativistic code to ascertain the stability and quality of the CPIFEL acceleration mechanism.

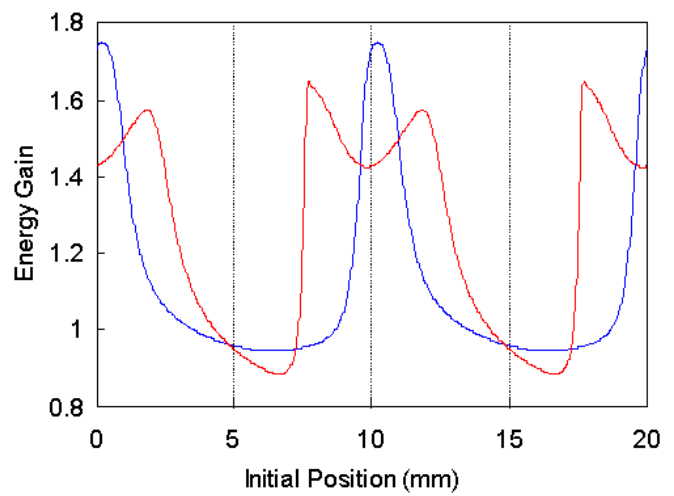

Figure 3. IFEL energy gain versus initial electron position in the wiggler.

\subsection{CPIFEL Energy Spectrum}

The results of a study of the acceptance of the IFEL accelerating bucket are shown in Fig. 3, where the energy gain is given as a function of the initial position in the wiggler, defined as the point where the laser pulse overtakes the electron. The periodicity is due to the 1-D nature of our model: the IFELA is invariant by translation of $\lambda_{w}$. Although both the drive laser pulse and the electron bunch are quite short, the nonlinear IFEL slippage length is a few centimeters, which explains how a ps electron bunch can be accelerated by a fs laser pulse: In the 1-D model, the phase of the ponderomotive force is determined by the initial electron position in the wiggler; because the IFELA is a fast-wave device, the acceptance of the accelerating bucket scales with the wiggler period. This should not be confused with the bunching which occurs at the IFEL resonant wavelength. The width of the energy peak in the chirped case, is seen to be nearly $2 \mathrm{~mm}$ FWHM. The fact that the IFELA bucket scale is given by the wiggler period directly results from the fast-wave nature of the IFELA interaction. This number is extremely large compared to the accelerating bucket in a laserplasma system, which is roughly equal to a tenth of the plasma wavelength (typically, in the micron range); this number is also compatible with conventional rf injectors, as is demonstrated in Fig. 4 (bottom). Here, we inject a 1ps FWHM Gaussian electron bunch at the optimum phase
$(0.2 \mathrm{~mm})$ for maximum energy gain, as shown in Fig. 4 (top); from the computed output electron energy spectrum, we find nearly $60 \%$ of the accelerated charge in a $0.125 \mathrm{MeV}$ bin at $57.8 \mathrm{MeV}$. This represents an energy spread of $<0.2 \%$; furthermore, $90 \%$ of the accelerated charge is found within a $0.5 \mathrm{MeV}$ energy interval, which corresponds to a spread smaller than $0.9 \%$.
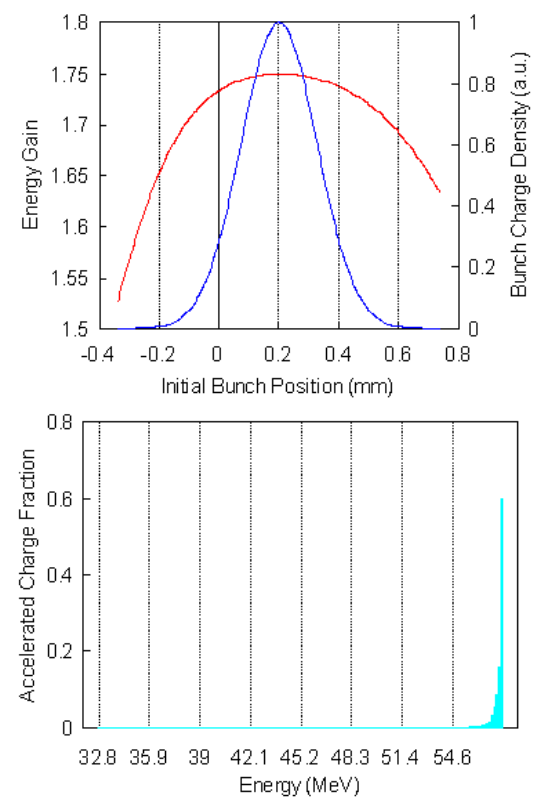

Figure 4. Top: initial electron bunch charge density and IFEL energy gain as a function of the initial position.

Bottom: IFEL output energy spectrum for a 1 ps FWHM Gaussian electron bunch injected at the optimum phase angle; the other parameters are the same as in Fig. 1.

\section{ACKNOWLEDGMENTS}

This work was partially supported under the auspices of the US Department of Energy by the Lawrence Livermore National Laboratory under Contract No. W-7405-ENG-48 through the Institute for Laser Science and Applications, and by the Department of Defense/Air Force Office of Scientific Research (MURI) F49620-95-1-0253, and ARO DAAHO4-95-1-0336.

\section{REFERENCES}

[1] I. Wernick and T. C. Marshall, Phys. Rev. A 46, 3566 (1992).

[2] A. Van Steenbergen, et al., Phys. Rev. Lett. 77, 2690 (1996).

[3] T. C. Marshall, Free-Electron Lasers (McMillan, New York, 1985); C. W. Roberson and P. Sprangle, Phys. Fluids B 1, 3 (1989).

[4] G. T. Moore, Phys. Rev. Lett. 60, 1825 (1988).

[5] M. D. Perry and G. Mourou, Science 264, 917 (1994).

[6] F. V. Hartemann, et al., Phys. Plasmas 6, 4104 (1999).

[7] D. Barnhart, Optica (Wolfram Research, Champaign, Illinois, 1995).

[8] C. P. J. Barty, et al., Opt. Lett. 19, 1442 (1994).

[9] F. V. Hartemann, et al., Phys. Rev. E 58, 5001 (1998). 